\title{
El yacimiento prehistórico de Jarama I (Valdesotos, Guadalajara). Campaña de prospección de 1991
}

\author{
Rogelio Estrada Garcia \\ Jesus F. Jordá Pardo * \\ Javier Pastor Muñoz
}

\section{INTRODUCCIÓN}

El yacimiento de Jarama I se encuentra situado en la margen derecha del rio Jarama, en el Término Municipal de Valdesotos (Guadalajara), a $820 \mathrm{~m}$ de altitud s. n. m. (figura 1, foto 1), dentro de la hoja n. ${ }^{\circ} 485$, Valdepeñas de la Sierra, del Mapa General (Serie L) a escala 1:50.000 de la Cartografia Militar de España.

La cavidad se desarrolla en las calizas del Cretácico Superior, que en esta zona se encuentran ligeramente plegadas. El sistema kárstico al que pertenece se encuentra atravesado por el rio Jarama, que discurre fuertemente encajado (foto 1), apareciendo en los acantilados de ambas márgenes gran cantidad de cuevas y abrigos rocosos, utilizados en ocasiones para el estabulamiento del ganado, algunos de los cuales contienen yacimientos arqueológicos dados ya a conocer (ADAN ÁlVAREZ et al., 1989; Adán Álvarez y Jordá Pardo, 1989; Jordá Pardo, 1985-1988, 1986; Jordá Pardo et al., 1988-1989, 1989; Jordá Pardo y Garcia VAlero, 1989). Por encima de las calizas cretácicas y de forma discordante, aparecen depósitos detriticos terciarios.

La cueva, orientada al SE, tiene pequeñas dimensiones debido a su destrucción parcial durante la construcción de la nueva carretera local que une Valdesotos con Puebla de Valles (láms. 1 y 2), si bien original-

* Instituto Tecnológico Geominero de España. Área de Ingenieria Geoambiental. 


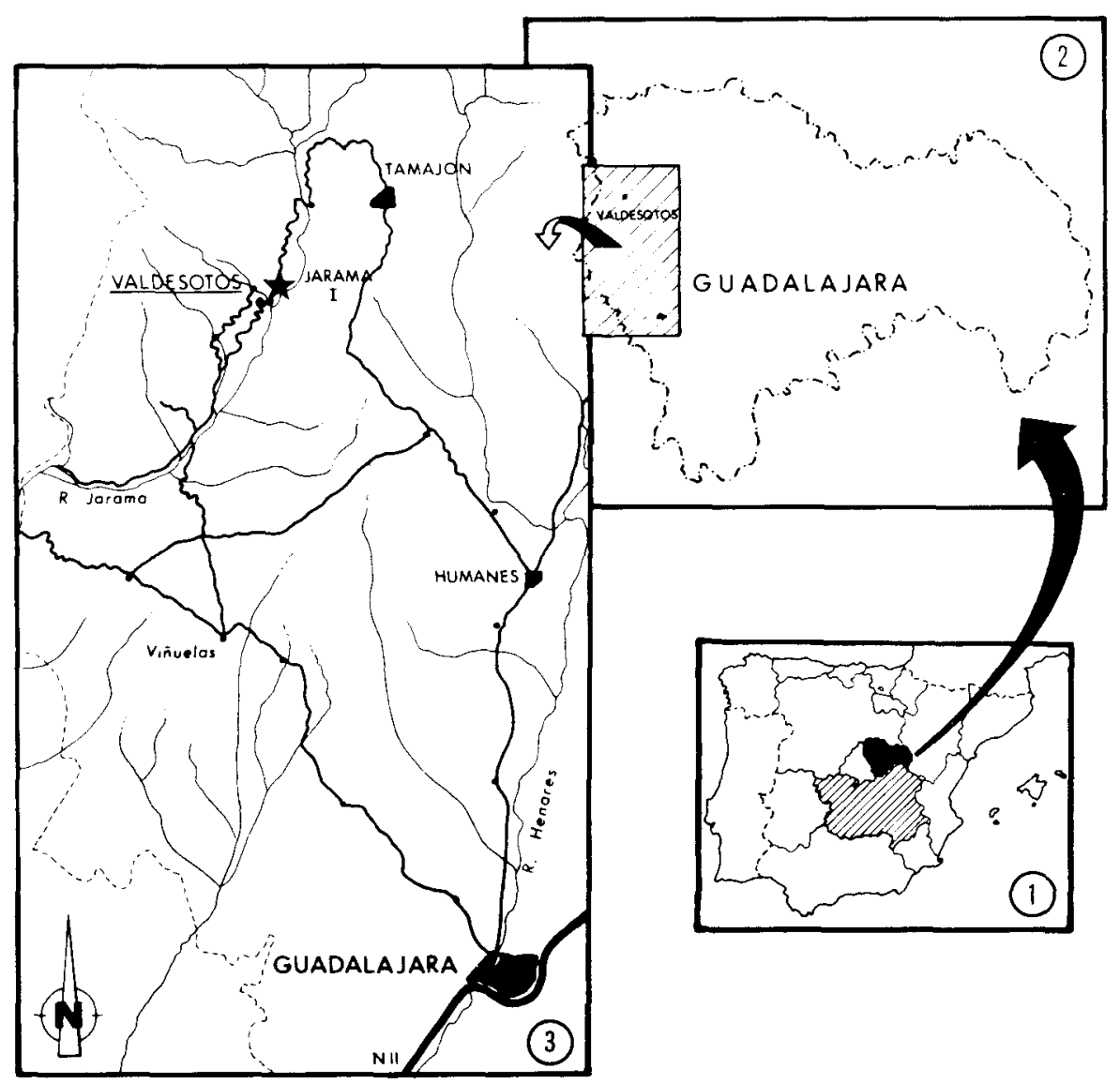

Fig. 1. Situación geográfica del yacimiento en el contexto peninsular (1), provincial (2) y local (3) (dibujo Federico Ramirez Trillo).

mente podria alcanzar unos $8 \mathrm{~m}$ de longitud máxima. El suelo presenta una topografía irregular y está formado por sedimentos fangosos de color rojo que contienen restos arqueológicos. La cavidad conserva zonas intactas en las que al menos existe una potencia sedimentaria superior a 1 m (lám. 2).

La cueva de Jarama I fue reconocida por técnicos arqueólogos del Museo Provincial de Guadalajara en 1982, durante las obras que propiciaron su parcial destrucción, quienes recuperaron materiales atribuidos a la Edad del Bronce, actualmente depositados en la citada institución. Posteriormente, en enero de 1983, el Prof. Dr. Francisco Jordá Cerdá y su 


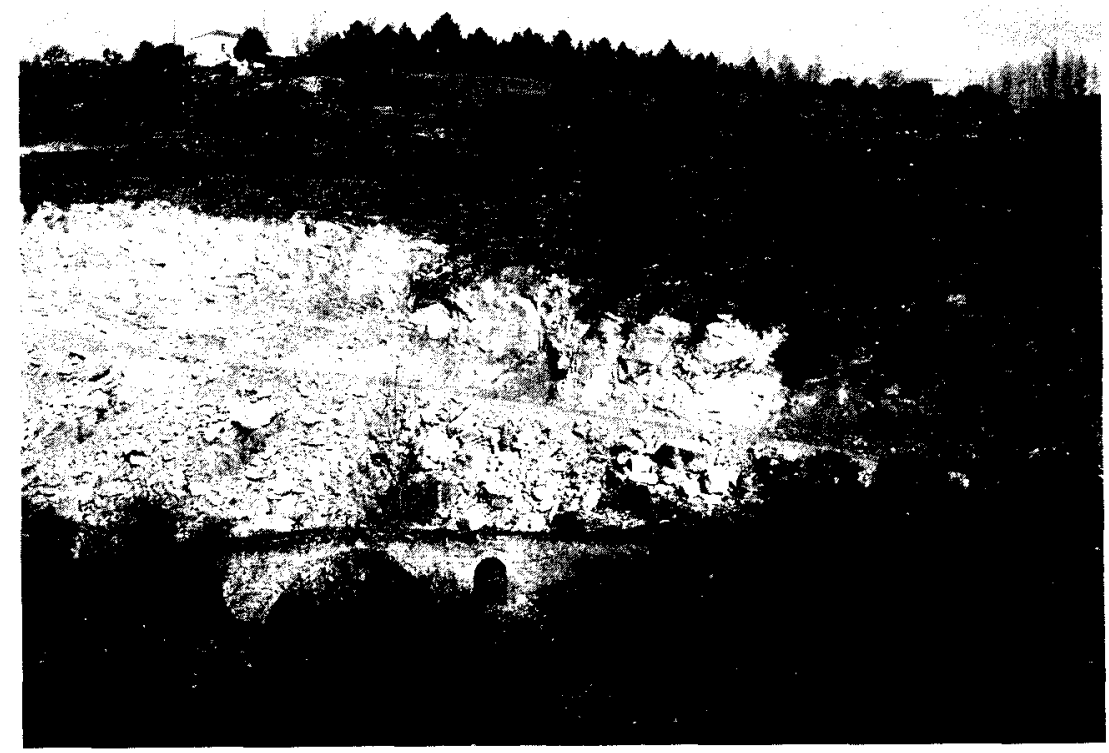

Lám. 1. Aspecto general del Alto Valle del Jarama en la zona del yacimiento.

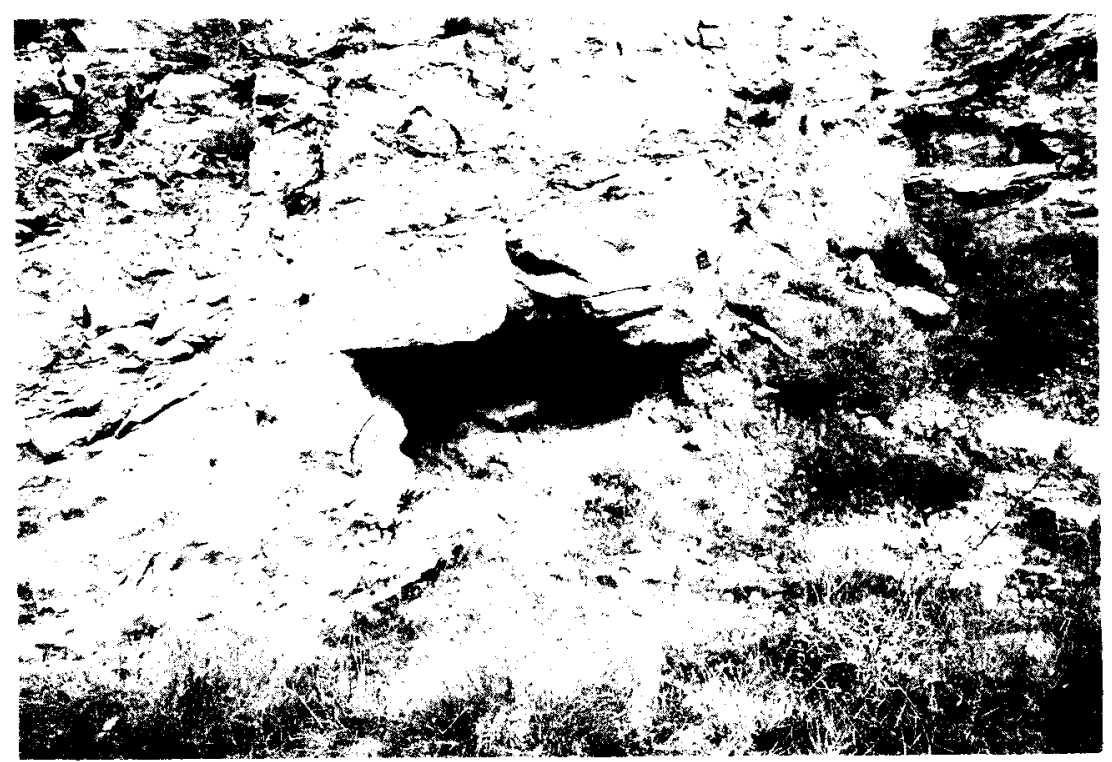

Lám. 2. Detalle del yacimiento de Jarama l mostrando los efectos de su destrucción parcial por las obras de la carretera y por la acción de clandestinos. 
equipo, en su Prospección Arqueológica del Alto Valle del Jarama, documentaron la existencia de un yacimiento paleolítico en la cavidad.

En este breve articulo damos a conocer los resultados de la Prospección Arqueológica en el yacimiento de Jarama I, realizada en 1991 bajo nuestra dirección (R.E.G. y J.F.J.P.), en el marco del Proyecto Investigaciones Prehistóricas en el Alto Valle del Jarama (Valdesotos, Guadalajara) (dirigido por J.F.J.P.), contando con el preceptivo permiso de la Consejeria de Educación y Cultura de la Junta de Comunidades de Castilla-La Mancha, que no estuvo acompañado de ninguna subvención económica.

La prospección se llevó a cabo en las numerosas visitas realizadas al yacimiento a partir de la obtención del Permiso, visitas realizadas con cierta periodicidad con el objeto de controlar las condiciones de conservación del yacimiento. En este sentido se constató que el mismo sufre saqueos parciales por parte de excavadores clandestinos, que realizan agujeros y zanjas en los sedimentos intactos (foto 2), lo que favorece su paulatina destrucción y la consiguiente pérdida irreparable de bienes del Patrimonio Histórico Español. En nuestra prospección, únicamente recogimos los materiales que se encontraban dispersos en superficie, producto de las remociones citadas.

Los objetivos con los que se planteó esta prospección fueron los siguientes: Recuperación de los materiales de superficie existentes en el yacimiento, producto de las actividades de clandestinos; valoración de los materiales recogidos para su correcta adscripción cronológica y cultural; comparación de las industrias recuperadas con las de los cercanos yacimientos de Jarama VI (Paleolítico Medio) y Jarama II (Paleolítico Superior); y finalmente, conservación y salvaguarda del patrimonio arqueológico que constituye el yacimiento de Jarama $I$.

A continuación expondremos brevemente las caracteristicas de los materiales recuperados, para finalizar realizando una valoración del yacimiento dentro del contexto geográfico y prehistórico del Alto Valle del Jarama.

\section{MATERIALES}

Los materiales arqueológicos recuperados componen una pequeña colección integrada por restos líticos, cerámicos y óseos.

Los restos cerámicos corresponden a tres fragmentos de galbo. Están realizados a mano, su pasta presenta abundantes desgrasantes de cuarzo 
de mediano y gran tamaño, siendo la cocción reductora. Las superficies de sus caras interna y externa se encuentran alisadas, sin que se aprecie ningún tipo de decoración. Estos fragmentos parecen corresponder con los recuperados en el yacimiento cuando fue parcialmente destruido por la construcción de la carretera local ya mencionada.

Los restos faunísticos recuperados se encuentran en un estado de conservación muy deficiente, predominando los fragmentos de huesos largos de macromamiferos. Actualmente estos restos se encuentran en estudio.

\subsection{Materiales líticos}

Antes de pasar a analizar el material lítico recuperado en Jarama I queremos resaltar que la parquedad de la muestra, asi como las condiciones del hallazgo, efectuado en el revuelto superficial, condicionan de forma drástica su análisis y consecuentemente las conclusiones que podemos inferir del mismo. No obstante, en las lineas que siguen trataremos de plasmar una visión que permita, en la medida de lo posible, aproximarnos a los caracteres generales de la industra lítica de Jarama I. Si bien, insistimos, las conclusiones de esta breve exposición deberán ser asumidas con las lógicas reservas dados los parámetros de trabajo en que nos movemos.

La pequeña colección recuperada se compone de 82 piezas líticas, realizadas en tres materias primas diferentes: cuarcita (12,19 por 100), cuarzo $(21,95$ por 100$)$ y silex $(65,85$ por 100). Con el objeto de efectuar un estudio más pormenorizado, hemos agrupado los diferentes restos liticos en función de las citadas materias primas.

\section{CUARCITA}

Esta materia prima es de mala calidad, de grano medio-grueso, y abunda en las inmediaciones del yacimiento. Este soporte les confiere a las piezas una marcada tosquedad, un engañoso aspecto arcaico, que contrasta con el carácter evolucionado que presenta la industria realizada en sílex. Algunas piezas poseen una fina pelicula calcárea que cubre parte de su superficie, originada por procesos de carbonatación postsedimentarios que tuvieron lugar en los depósitos del yacimiento.

\section{Núcleos (3 ejemplares)}

Dos de estos núcleos son de tipología amorfa. Uno de ellos es un canto de grandes dimensiones $(111 \times 116 \times 63 \mathrm{~mm})$ en el cual se efectuaron algunas extracciones primarias de lascas de gran tamaño. 
El tercero es una interesante pieza que en origen fue una espesa lasca de decorticado primario, con talón cortical, acondicionada posteriormente como núcleo $(93 \times 80 \times 33 \mathrm{~mm})$. Presenta algunas extracciones en el extremo distal de su cara ventral. Su dorso se encuentra acondicionado mediante extracciones centripetas en su contorno, salvo en el extremo distal, donde presenta cortex, que prefiguran una plataforma de golpeo de la cual se extrajo una lasca. Se trata por tanto de un tipo cuya técnica de talla es claramente Levallois.

Restos de talla (7)

Se trata de grandes piezas de desbastado, con talones corticales (4) y lisos (3). Son: tres lascas de decorticado primario, dos lascas de decorticado secundario y dos lascas simples.

\section{CUARZO}

Si bien pueden constatarse claras huellas de trabajo en las piezas que utilizan como soporte el cuarzo, parece inferirse de la muestra analizada, que este no permitía excesivos alardes técnicos, salvo en el caso del cristal de roca, dada la deficiente calidad del cuarzo utilizado. Sin embargo, debido a su abundante presencia en el sustrato litológico de la zona, su explotación es ciertamente relevante en el yacimiento. En su mayoría, estas piezas presentan una fina pelicula calcárea en la superficie.

\section{Útiles (1)}

Buril diedro de ángulo, sobre hoja, realizado en cristal de roca (34 $\mathrm{x}$ $17 \times 5 \mathrm{~mm})$.

Núcleos (3)

Se trata de piezas de pequeño tamaño: uno, globular sobre canto de cristal de roca que presenta abundantes extracciones, y dos amorfos.

Restos de talla (14)

Salvo las lascas de decorticado primario, son fragmentos de pequeño tamaño y morfologia muy irregular: tres lascas de decorticado primario, diez lascas simples (una en cristal de roca) y un chunk.

\section{SILEX}

Como apuntábamos anteriormente, la industria realizada en sílex presenta un aspecto evolucionado, producto de una etapa avanzada en el 
proceso de talla, con un predominio claro de las lascas y hojitas de pequeño tamaño. Junto a este fuerte aprovechamiento de la materia, como denota el análisis del sustrato de la industria, destaca la ausencia de núcleos en el conjunto, producto quizás de aquella caracteristica. La mayoria de las piezas sufren una incipiente desilicificación, presentando asi mismo una fina pelicula calcárea en buena parte de sus superficies. En dos casos se aprecia un alteración de tipo térmico.

Útiles (6)

Raspador simple (figura 2.4) sobre lasca. Presenta el frente parcialmente fracturado por golpes perpendiculares al mismo $(32 \times 29 \times 7 \mathrm{~mm})$.

Buril diedro de ángulo $(37 \times 13 \times 8 \mathrm{~mm})$ (figura 2.6).

Buril nucleiforme $(54 \times 21 \times 12 \mathrm{~mm})$.

Hoja con retoque contínuo sobre un borde con pequeño retoque parcial en el extremo basal del borde opuesto $(74 \times 18 \times 8 \mathrm{~mm})$ (figura 2.1). Se trata de un retoque semiabrupto escamoso similar al tipico auriñaciense. En esta pieza se puede apreciar a simple vista un desgaste parcial del filo retocado.

Pieza astillada $(28 \times 28 \times 11 \mathrm{~mm})$.

Diverso. Fragmento distal de hoja (figura 2.2) con pequeños retoques, quizás seudorretoques, parciales en uno de los bordes de la cara dorsal $(46 \times 17 \times 5 \mathrm{~mm})$.

Crestas, hojas y hojitas (17)

Se recogió un porcentaje elevado de estas piezas si se compara con el resto de la industria elaborada en silex. Son: cinco hojas, 11 hojitas y una cresta.

Restos de talla (31)

Se trata en general de lascas de pequeño tamaño con talones lisos en su mayoria. Son: una lasca de decorticado primario, 25 lascas simples y cinco debris.

\subsection{Caracteristicas generales de la industria}

En cuanto a la materia prima, cabe reseñar, la marcada explotación diferencial de la misma. Existe un predominio claro en la utilización del sílex (de distintos tipos), junto a un uso preferencial de esta roca en la 

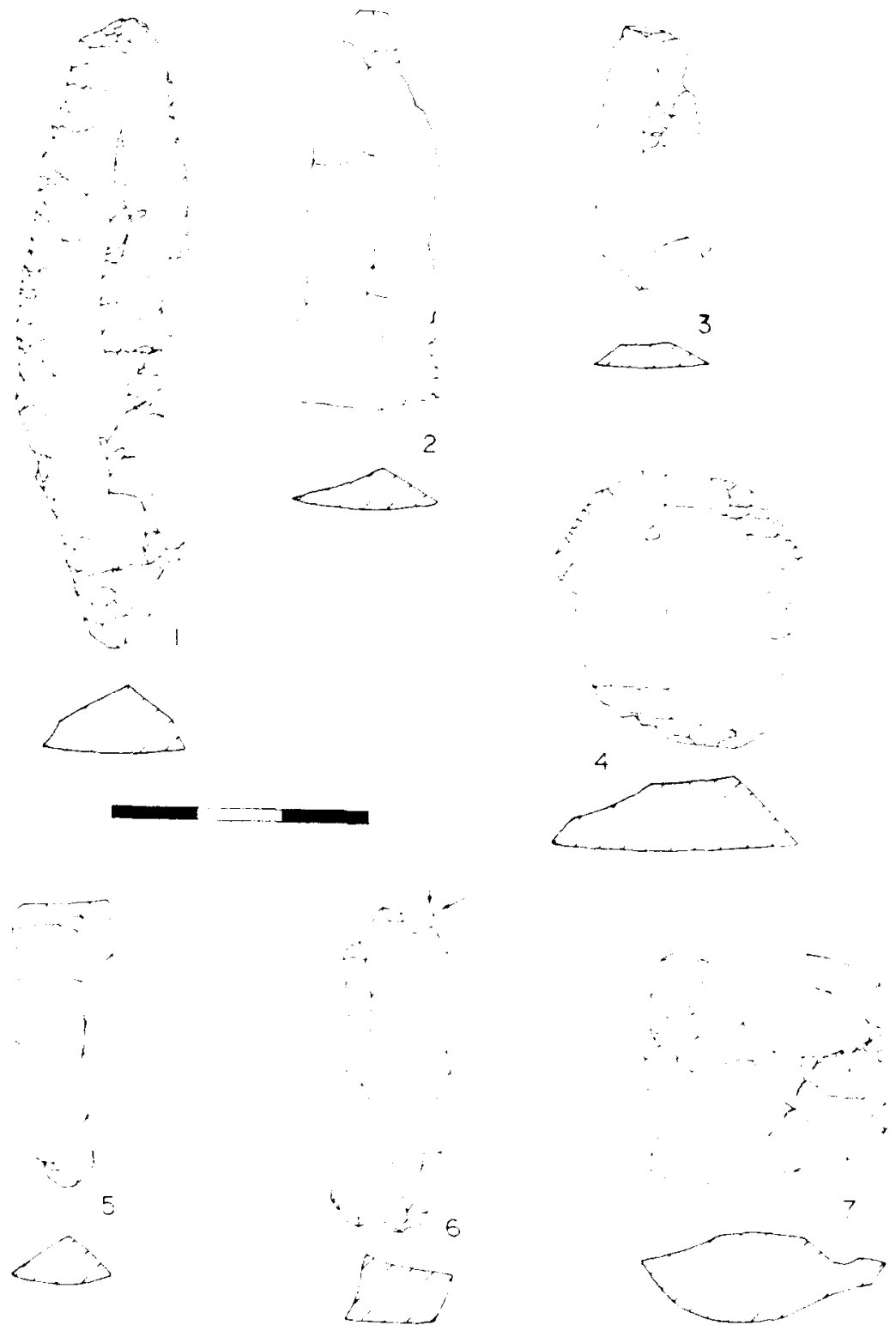

Fig. 2. Industria litica de Jarama l: 1, hoja con retoque contínuo, 2, fragto. distal de hoja, 3, hoja, 4, raspador simple, 5, fragto proximal de hoja, 6, buril diedro, 7 , pieza astillada (dibujo Javier Pastor Muñoz). 
elaboración de útiles. Este hecho es ciertamente relevante si tenemos en cuenta la ausencia de silex en este tramo de la Cuenca del Jarama. Las áreas de aprovisionamiento más cercanas se situarian en las cuencas de los rios Sorbe y Henares, unos $20 \mathrm{~km}$ al E de nuestra zona.

La cuarcita y el cuarzo (incluido el cristal de roca) presentan un menor grado de aprovechamiento, en líneas generales. En el caso del cuarzo se recogieron pequeñas lascas, producto en parte de una defectuosa talla, dada la dificultad que plantea su trabajo. En ocasiones, sin embargo, cuando el cristal de roca es de buena calidad, se aprecia también un mayor aprovechamiento, como es el caso de un núcleo o de un buril. Estos materiales se encuentran en las inmediaciones del yacimiento, bien en forma de cantos arrastrados por el río, bien en los afloramientos de las rocas que componen el basamento de las sierras de Somosierra y Ayllón.

Otra característica destacable es el elevado porcentaje de industria laminar realizada en silex. Salvo contadas excepciones, que parecen obedecer más al tipo de materia prima utilizada que a una caracteristica derivada de la tradición industrial, se aprecia en el lote una clara tendencia leptolítica. Pese a lo reducido de la muestra, cabe destacar también el predominio de los buriles dentro de los útiles.

\section{VALORACIÓN DEL YACIMIENTO Y CONSIDERACIONES FINALES}

El yacimiento de Jarama I se encuentra situado en el Alto Valle del Jarama, en una pequeña cavidad abierta en las calizas cretácicas del borde meridional de las sierras de Somosierra y Ayllón.

En este valle, los trabajos de prospección arqueológica llevados a cabo por nosotros desde 1983 nos han permitido localizar varios yacimientos. Uno de ellos, Jarama 11 , ha sido ya excavado, localizándose en él restos correspondientes al Magdaleniense Inferior (ADAN ÁLVAREZ et al., 1989; AdAn Álvarez y Jordá Pardo, 1989; Jordá Pardo, 1985-1988, 1986; JORDA PARDo et al., 1989) entre los que destaca una estatuilla de marfil que representa un glotón (JORDÁ PARDo y GARCIA VALERO, 1989; JORDÁ PARDo et al., 1988-1989), asi como un enterramiento de la Edad del Bronce. Otro de los yacimientos, Jarama VI, se encuentra actualmente en proceso de excavación, reconociéndose hasta el momento tres niveles de ocupación del Paleolitico Medio incluidos dentro de una dinámica sedimentaria muy interesante. 
En el caso de Jarama I hemos podido documentar hasta la fecha la presencia de materiales de la Edad del Bronce, junto con una interesante colección de materiales líticos de clara filiación paleolítica.

Este último conjunto, recogido en superficie y carente de todo contexto estratigráfico, presenta un escaso número de piezas, destacando la ausencia de industria ósea y de elementos líticos claramente diagnósticos. Por esto, resulta un tanto aventurado determinar la adscripción cultural del conjunto, si bien, las características generales que presenta nos permitirian situarlo en las etapas finales del Paleolítico Superior, probablemente en algún momento del periodo Magdaleniense. La presencia en torno al Sistema Central de yacimientos atribuidos a este mismo periodo con industrias elaboradas en cuarzo y cristal de roca (FABIÁN, 1984-1985, 1985) confiere a nuestra hipótesis un mayor grado de fiabilidad.

De confirmarse la veracidad de esta hipótesis, la futura excavación sistemática de este yacimiento (Jarama I) nos permitirá establecer una correlación con el de Jarama II, situado a una distancia inferior al centenar de metros. En este sentido cabe destacar que el yacimiento de Jarama II presenta unas caracteristicas negativas en cuanto a su habitabilidad, con un difícil acceso y una mala orientación a la radiacción solar, que parece sugerir, junto con los materiales que contiene, su posible utilización con fines rituales (AdÁn ÁlVAREZ et al., 1989; AdÁ ÁlVAREZ y JordÁ Pardo, 1989; Jordá Pardo, 1985-1988, 1986; Jordá Pardo et al., 19881989, 1989; JordÁ Pardo y Garcia Valero, 1989).

Por el contrario, Jarama I presenta unas caracteristicas óptimas en cuanto a la habitabilidad (amplitud, fácil acceso, excelente orientación, proximidad al rio, control de abrevaderos y zonas de paso de animales, etcétera) que permitirian la existencia de una ocupación intensa y prolongada, dentro de un espacio habitacional relativamente extenso.

Actualmente, se puede apreciar en el fondo de la cavidad (única parte que se conserva de ésta), una secuencia estratigráfica inalterada de aproximadamente $1,5 \mathrm{~m}$ de espesor, a cuyo techo pertenecen los materiales recogidos en esta prospección. Estos depósitos se conservan en una extensión de unos 10 metros cuadrados, por lo que su excavación contribuiria enormente al conocimiento de las caracteristicas tecnoculturales y ambientales del yacimiento. La excavación arqueológica sistemática de Jarama I, junto con la ya realizada en Jarama ll y a la que se lleva a cabo en Jarama VI, permitiria llegar al conocimiento exhaustivo de las ocupaciones humanas y del medio ambiente del Pleistoceno Superior del Alto Valle del Jarama.

Este hecho es ciertamente relevante, dado que en la actualidad, el planteamiento del Proyecto Investigaciones Prehistóricas en el Alto Valle 
del Jarama permite una aproximación global e interdisciplinar al conocimiento del hábitat humano durante un periodo de tiempo amplio y en un espacio determinado, en una zona de la Península donde este tipo de estudios no se han llevado a cabo hasta la fecha.

Los resultados de este proyecto interdisciplinar permitirán establecer un marco de referencia sólidamente elaborado, sobre el cual se podrán contrastar las futuras investigaciones (excavaciones de urgencia y sistemáticas, prospecciones, hallazgos aislados) que se realicen sobre estas etapas de la Prehistoria hasta ahora prácticamente desconocidas en la Comunidad de Castilla-La Mancha.

\section{RESUMEN}

Los materiales líticos recogidos en la prospección superficial llevada a cabo en Jarama I componen una colección integrada por lascas y hojas, núcleos y diversos materiales retocados (raspadores, buriles, hojas retocadas, etc.). Las materias primas son sílex, cuarzo, cuarcita y cristal de roca. Estos materiales se pueden adscribir al Paleolitico Superior. También se han recuperado algunos fragmentos cerámicos que se pueden atribuir a la Edad del Bronce. Además se ha constatado un cierto número de restos óseos de macromamiferos.

\section{REFERENCIAS}

Adan Álvarez, G. Garcia Valero, M. A., Jorda Pardo, J. F. y Sanchez Chillon, B. (1989): “Jarama II, nouveau gisement Magdalénien avec art mobilier de la Meseta Castellana (Guadalajara, Espagne)", Bulletin de la Société Préhistorique de IÁriège, t. XLIV, págs. $97-120$.

Adan Álvarez, G. y Jorda Pardo, J. F. (1989): «Industrias óseas del Paleolitico y Postapaleolítico pirenaico en relación con los nuevos hallazgos de Jarama II (Guadalajara)", Espacio, Tiempo y Forma, Serie 1, 2, págs. 109-130. Madrid.

FABIAN, F. (1984-1985): "Los útiles de arista diédrica sobre prismas piramidales o nódulos de cristal de roca (U.A.D.) en el yacimiento de La Dehesa, El Tejado de Béjar (Salamanca). Estudio morfotécnico», Zephyrvs, XXXVII-XXXVIII, págs, 115-124.

- (1985): “El Cerro del Berrueco. Cási diez mil años de habitación ininterrumpida". Revista de Arqueologia, n." 65, págs. 6-17. Madrid.

Jorda PARdo, J. F. (1985-1988): "Investigaciones Prehistoricas en el Alto Valle del Jarama (Guadalajara)", I Congreso de Historia de Castilla-La Mancha, t. II, Pueblos y Culturas Prehistóricas y Protohistóricas (1), págs. 111-123.

- (1986): «Jarama II. Nuevo yacimiento del Paleolitico Superior», Revista de Arqueologia, n. ${ }^{\circ}$ 61, págs. 14-24. Madrid. 
Jorda Pardo, J. F., Garcia Valero, M. A., Perez Morales, C., Sanchez-Monge llusa, M., Estrada Garcia, R., Benito F. y Sanchez Chillon, B. (1989): "Investigaciones Prehistóricas en el Alto Valle del Jarama", Revista de Arqueologia, n. "94, págs. 61-62. Madrid.

JoRdA PARDO J. F. y GARCiA VALERO, M. A. (1989); "Las representaciones de glotón (Gulo gulo L) en el Arte Paleolitico pirenaico y un nuevo hallazgo de arte mueble en el Alto Valle del Jarama (Guadalajara)", Espacio, Tiempo y Forma, Serie I, 2, págs. 89-105. Madrid. Jorda Pardo, J. F., Garcia Valero, M. A., Adan Álvarez, G. y Sanchez Chillon, B. (1988-1989): "Una nueva pieza de arte mueble magdaleniense: el glotón de la Cueva de Jarama II (Valdesotos, Guadalajara)», Ars Praehistórica, VII-VIII, págs. 107-122 (in lit.). 